\title{
Design of a robotic instrument for minimally invasive waterjet surgery
}

\author{
Christopher Schlenk \\ Institute of Robotics and Mechatronics \\ German Aerospace Center (DLR) \\ Oberpfaffenhofen, Germany \\ christopher.schlenk@dlr.de
}

\author{
Thomas Bahls \\ Institute of Robotics and Mechatronics \\ German Aerospace Center (DLR) \\ Oberpfaffenhofen, Germany \\ thomas.bahls@dlr.de
}

\author{
Andrea Schwier \\ Institute of Robotics and Mechatronics \\ German Aerospace Center (DLR) \\ Oberpfaffenhofen, Germany \\ andrea.schwier@dlr.de
}

\author{
Michael Heiss \\ Institute of Robotics and Mechatronics \\ German Aerospace Center (DLR) \\ Oberpfaffenhofen, Germany \\ michael.heiss@dlr.de
}

\author{
Alin Albu-Schäffer \\ Institute of Robotics and Mechatronics \\ German Aerospace Center (DLR) \\ Oberpfaffenhofen, Germany \\ alin.albu-schaeffer@dlr.de
}

\begin{abstract}
Using a pressurized waterjet for cutting or abrasing tissue is a well established method in surgery. This paper presents a robotic tool for minimally invasive waterjet surgery with two degrees of freedom, integrated suction and an optional splash protection. The function of the tool and the effect of the splash protection on the suction of the applicated water was successfully evaluated in tests with ballistic gelatine. Based on this evaluated design, a concept for further increasing the oscillation frequency of the instrument tip is introduced and the results of preliminary tests of a simplified mockup are shown.
\end{abstract}

Index Terms-Medical robotics, instrument, waterjet, surgery

\section{INTRODUCTION}

Using waterjet instruments for surgical interventions allows a selective cutting or abrasing of patient tissue. By changing the water pressure the abrasiveness of the waterjet can be adapted to the surgical needs. In liver surgery, for example, it is thereby possible to remove the soft intrahepatic parenchyma while preserving the ducts and vessel lying within the parenchyma. This simplifies the hemostasis and reduces the blood loss of the patient [1]-[6]. Further applications of waterjet instruments, which have to date been investigated, include debridement of chronic wounds [7] and open fractures [8], kidney surgery [9], treatment of rectum cancer [10], removal of lymphatic tissue in testis-cancer patients [11] and neurosugery [12]-[14]. While in earlier studies adapted agricultural sprayers [1] or specially designed pumps and handpieces [2], [3] had to be used, several waterjet systems are commercially available today - e.g. the ERBEJET 2 [Erbe Elektromedizin GmbH, Tübingen, Germany], the Debritom [Medaxis AG, Baar, Switzerland] and the Versajet II [Smith \& Nephew plc, London, UK] [15]-[17]. The application possibilities of these systems, however, are currently limited by

The presented work was funded by the Helmholtz Association as part of the MIRO Innovation Lab (program name: HIL-03 MIRO Lab). 978-1-53867825-1/19/\$31.00 @2019 IEEE the available handheld, mostly rigid applicators. While these applicators are sufficient for open surgery, their handling in minimally invasive surgery is cumbersome, especially if not only cutting but abrasion of tissue is required: Firstly, due to the loss of two degrees of freedom (DoF) in the trocar, it is not always possible to orientate the waterjet from the applicator orthogonal to the tissue surface. This may reduce the abrasive effect and complicate suction. To allow an orthogonal orientation of the applicator tip and the tissue also in situations when the trocar axis is not orthogonal to the tissue, an intracorporal wrist with at least one DoF is necessary. Secondly, the spatial abrasion of tissue requires many precise, repeated applicator tip motions, which are laborious to perform with an handheld instrument. Thirdly, the splashing of the pressurized liquid (typically physiological saline solution [1], [2]) after hitting the tissue, obscures the endocopic image.

To overcome these limitations, we present in the following a robotic instrument for minimally invasive waterjet surgery providing a universal wrist with two DoF and a splash protection made from highly flexible silicone. The setup of the robotic system consisting of the robotic instrument and the robot arm DLR MIRO is displayed in figure 1.

\section{Materials AND Methods}

Within this study, an ERBEJET 2 has been used as pump and suction unit and a commercial flexible probe (Erbe Item No. 20150-020) with an outer diameter of $d_{W j}=1.3 \mathrm{~mm}$ and a nozzle diameter of $120 \mu \mathrm{m}$ as waterjet applicator.

The design of the instrument is based on the instrument for minimally invasive robotic surgery (MIRS) developed by THIELMANN ET AL. [18]. While the versatile drive unit is used without modifications, the autoclavable tool was adapted for the integration of the waterjet probe and the suction hose. The revised tool (see figure 2) provides a universal wrist joint with two DoF close to the tool tip, which is actuated by the versatile drive unit via two tendons in the tool shaft. Each 


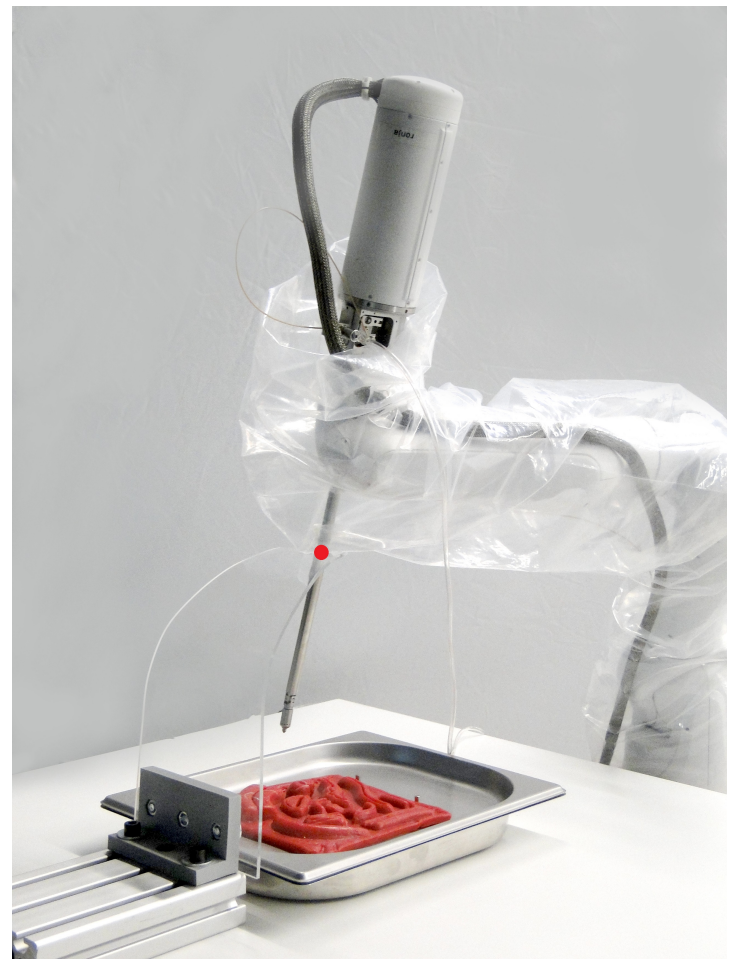

Fig. 1. Total view of the robotic system for waterjet surgery. The instrument, consisting of versatile drive unit and waterjet tool is attached to the instrument interface of the robot arm DLR MIRO. Thanks to the universal joint near the tool tip, the axis of the waterjet nozzle can be orientated orthogonal to the tissue phantom while maintaininig the trocar point (red dot).

tendon features two crimped balls at its both ends and one crimped ball at its center. The crimped balls at the centers of the both tendons are pressed in two round cavities in the distal part of the universal joint (one of these cavities can be seen in Fig 2 c)). More details about the function of the universal joint are revealed in the paper by THIELMANN ET AL. in figure 4. Instead of the orange and green ring shown in this schematic figure, the both ends of the tendons are connected to a system of levers and pulleys in the tool interface of the real system. The two actuated DoF of the universal joint offer for the following advantages:

- More effective cutting: The cutting/abrasion effect is maximum when the impact pressure of the waterjet can be used. As soon as a water film in the target area has evolved, only the less effective stagnation pressure acts on the tissue. To ensure that the waterjet affects areas without water film, the instrument tip must perform a high-frequency oscillating motion [19], [20]. BAHLS ET AL. proposed an approach to automatically calculate these high-frequency motion with small amplitude, the transection, around the user controlled or automatically planned macroscopic motion, the dissection. By using Lissajous curves for defining the transection trajectories, several kinds of continuous 1-D and 2-D figures can be created by varying just four parameters [20]. The waterjet instrument tip must move with high frequencies in one dimension to follow the transection trajectories for cutting and in two dimensions to follow the transection trajectories for abrasion. If in a robotic application the instrument was rigid, the transection would have to be realized by motions of the robot arm. Moving the whole mass of the robot at high frequencies, however, would result in a significant load on the robot's drive train with undesirable consequences like noise and increased wear of mechanical components. In contrast, in an instrument with - at least - two actuated DoF, only these must be moved to follow the transection trajectories. The DoF of the robot arm are only used to realize the slower dissection motion. Due to the small mass of the instrument tip distal from the two instrument DoF, the resulting load for the instrument drive unit is comparatively low.

- Simplified planning for minimally invasive interventions: Due to the motion capabilities of the wrist joints the instrument shaft does not have to be orthogonal to the tissue surface to enable an orthogonal orientation of the nozzle axis w.r.t. the tissue surface. This gives the surgeon more freedom in choosing the trocar position and thereby simplifies the preoperative planning of the minimally invasive intervention [4], [19].

The integration of the suction within the waterjet applicator renders a separate suction device unnecessary. Thus the sight of the surgeon on the operation field is improved and - in minimally invasive surgery - the necessary number of trocars is reduced.

The addition of the splash protection has two main objectives: Firstly the splash protection shall reduce the amount of water splashed by the waterjet to prevent the obstruction of the endoscopic view by water drops on the endoscope lense and mist around the operation field. Secondly it shall improve the effectiveness of the suction. To do so, it must be moved close to the tissue surface. To minimize the risk of tissue damage in case of collisions, the splash protection must be highly flexible. At the same time its material must be sterilizable and preferably transparent to allow visual control of the waterjet application.

The function of the designed robotic instrument for minimally invasive waterjet surgery was evaluated in two types of tests:

- Function tests of the suction: The time necessary for sucking $100 \mathrm{ml}$ of tap water from a measuring glass was stopped once for a wrist joint deflection of $0^{\circ}$ and once for a deflection of $35^{\circ}$. Subsequently the tool was investigated for leaked water and the average suction speed for both cases was calculated.

- Function tests of the splash protection: For testing the effect of the splash protection on the cutting and suction performance, phantoms made of the ballistic gelatine GELITA GELATINE Type Ballistic 3 [GELITA AG, Eberbach, Germany] in a $10 \%$ ratio gelatine : water were used. According to JUSILLA ET AL. hydrogels with 10-20 $\%$ gelatine are typically used to simulate the behavior of 

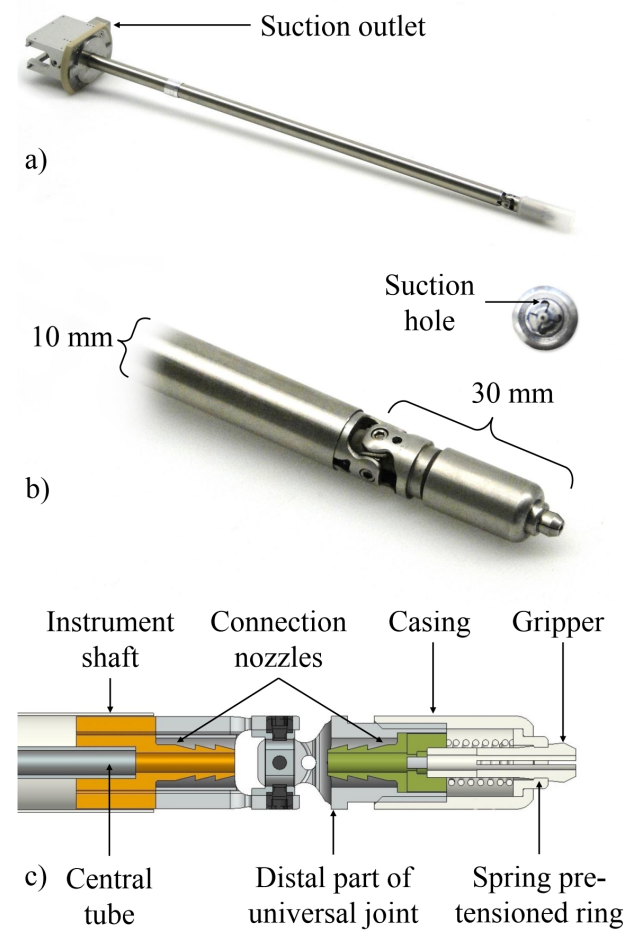

Fig. 2. a) View of the complete waterjet tool with the tool interface on the left and the silicone splash protection on the right.

b) Detail view of the tool tip without splash protection. The suction is carried out through four holes equally distributed around the spring pretensioned ring. c) Cross section of the proximal part of the waterjet instrument without splash protection (for clarity without flexible probe, suction hose and actuation tendons): The tip of the flexible probe is clamped in the slotted part of the gripper, which is compressed by the spring pretensioned ring. The pretension can be adapted by screwing the casing with more or less overlap on the distal part of the universal joint. The hose of the flexible probe is routed through the center of the universal joint and the central tube of the instrument shaft. Since the outer diameter of the flexible probe is smaller than the inner diameter of the central tube, the remaining cross section can be used for suction. To flexibly connect the suction channels on the proximal and the distal side of the universal joint, a EPDM/PP hose is slided on the connection nozzles and on both sides of the joint.

muscle tissue [21]. The applied phantoms therefore mimic the behavior of soft muscle tissue. The waterjet nozzle was moved orthogonal to the surface of these phantoms on a rectangular meander with an amplitude of $100 \mathrm{~mm}$ and a distance between the amplitudes of $4 \mathrm{~mm}$. During each test, 15 rectangular meanders (with 30 straight, 100 $\mathrm{mm}$ long lines) were completed. The waterjet was running during the whole test with a pressure of 35 bar, the suction with a pressure of -800 mbar. The influence of the distance between waterjet nozzle and phantom surface on the suction performance was investigated by testing four different distances: $4 \mathrm{~mm}, 6 \mathrm{~mm}, 8 \mathrm{~mm}$ and $10 \mathrm{~mm}$. For each distance, one test run was performed without and one with splash protection (the distance between splash protection and phantom surface was $1 \mathrm{~mm}$ ). Subsequently the ratio between water removed by the integrated suction and applicated water was calculated for both scenarios.

\section{RESULTS}

The developed waterjet tool provides the following features:

- Autoclavable: The tool contains no electronic components and consists completely of autoclavable materials like stainless steel, aluminum, EPDM/PP and silicone to facilitate its sterilization.

- Clamping mechanism for flexible probe: A spring preloaded clamping mechanism (compare figure 2) allows the tool-free change of the flexible probe (e.g. when the nozzle is blocked). To remove the probe, the overlap of the casing and the distal part of the universal joint must be increased by screwing the casing on the distal part. Thus the spring pretensioned ring is moved towards the universal joint and the load on the slotted part of the gripper is reduced. Now the probe can easily be exchanged. Subsequently the casing overlap of casing and distal part of the universal joint is reduced again to restore the clamping between gripper and probe.

- Compact physical dimensions: As displayed in figure $2 \mathrm{~b}$ ), the waterjet tool without the splash protection fits through a standard $10 \mathrm{~mm}$ trocar. The distance between the axes of the joint and the waterjet nozzle is $30 \mathrm{~mm}$.

- Two DoF in a universal joint: The universal joint allows a rotation of $\pm 35^{\circ}$ around both rotation axes [18]. Since the flexible probe and the suction hose pass through the center of the universal joint, their strain due to the bending of the wrist is minimal.

- Integrated suction: Four suction holes are distributed around the spring pretensioned ring. The pump is connected via a flexible hose to the outlet at the tool interface. The fluid is guided through the central tube and an EPDM/PP suction hose with inner diameter $D_{i}=2.54$ $\mathrm{mm}$ and outer diameter $D_{a}=4.22 \mathrm{~mm}$ [RCT Reichelt Chemietechnik GmbH + Co., Heidelberg, Germany], which is used to flexibly connect the suction channels on the proximal and the distal side of the universal joint. The hose of the waterjet probe with an outer diameter of $d_{W j}=1.3 \mathrm{~mm}$ is surrounded by the hose for the integrated suction. Therefore the cross-sectional area of the suction can be calculated as:

$$
A_{S}=\pi\left(\frac{D_{i}^{2}}{4}-\frac{d_{W j}^{2}}{4}\right)=3.74 m^{2}
$$

The function tests of the suction proved the leak tightness of the suction and revealed a suction speed of $5.9 \pm$ $0.15 \mathrm{ml} / \mathrm{s}$ for the elongated wrist joint (number of tests $\mathrm{n}=3$ ) and $5.7 \pm 0.42 \mathrm{ml} / \mathrm{s}$ for the wrist joint tilted by $35^{\circ}$ around one axis (number of tests $n=4$ ).

- Flexible splash protection: To meet the requirements regarding flexibility, sterilizability and transparency, the splash protection with a wall thickness of $0.6 \mathrm{~mm}$ (see figure 3) was molded in a 3D-printed cast from the silicone Dragon Skin 30 [Smooth-On Inc., Macungie, PA, USA], which has a shore hardness of $30 \mathrm{~A}$. Using the splash protection reduced the ratio between water removed by the integrated suction and applicated water 

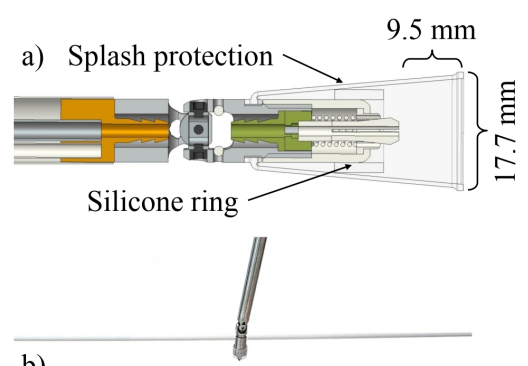

b)
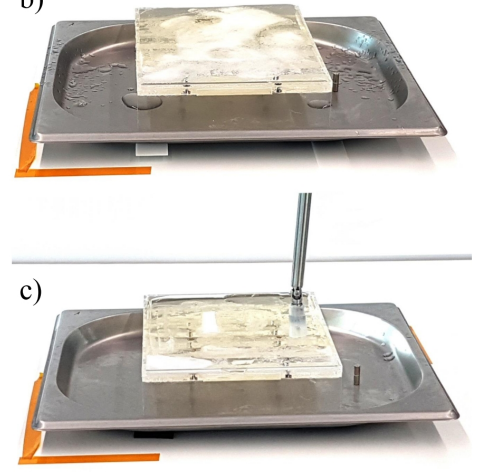

Fig. 3. The final version of the splash protection (a)) consists of the conic, molded splash protection on the outside and a ring of stiffer silicone between splash protection and instrument stucture.

Effect of the splash protection for a distance of $6 \mathrm{~mm}$ : Without splash protection (b)) the bowl with the gelatine is filled with water and a significant amount of water has splashed outside the bowl. With the splash protection (c)) the remaining amount of water is smaller and almost no water splashed outside the bowl.

in all performed tests (compare table I). In the first test with splash protection (distance $10 \mathrm{~mm}$ ) it was observed, that a collision of the splash protection with the phantom surface during active suction caused a momentary collapsing of the splash protection around the tooltip and thus an interruption of the waterjet cut. The addition of a silicone ring (inner diameter $8 \mathrm{~mm}$, outer diameter 11 $\mathrm{mm}$, shore hardness $60 \mathrm{~A}$ ) between tool tip and the splash protection resolved this problem. The final structure of the splash protection and its effect on the suction and the avoidance of splashing is displayed in figure 3 .

TABLE I

RESULTS OF THE FUNCTION TESTS OF THE SPLASH PROTECTION: RATIOS BETWEEN WATER REMOVED BY THE INTEGRATED SUCTION AND APPLICATED WATER. FOR EACH TEST SCENARIO ONE TEST RUN (FIFTEEN RECTANGULAR MEANDERS WITH AN AMPLITUDE OF 100 MM AND A DISTANCE BETWEEN THE AMPLITUDES OF 4 MM) WAS CONDUCTED.

\begin{tabular}{|c|c|c|}
\hline Distance [mm] & Without splash protection & With splash protection \\
\hline 4 & $63.17 \%$ & $82.26 \%$ \\
6 & $50.14 \%$ & $85.84 \%$ \\
8 & $31.55 \%$ & $89.68 \%$ \\
10 & $5.21 \%$ & $76.18 \%$ \\
\hline
\end{tabular}

\section{DISCUSSION}

The designed tool and the versatile drive unit for its actuation can be attached to the versatile robot arm DLR MIRO to perform both open and minimally invasive robotic waterjet surgery. The feasibility of the approach has been demonstrated in phantom studies using ballistic gelatine [19], [20] as well as in ex vivo studies on porcine skin tissue [22]. Due to the larger diameter of the suction hose compared to the combination of tendon and spring in the original DLR MICA tool [18], the range of motion of the universal joint is slightly reduced: Instead of $40^{\circ}$ the universal joint of the waterjet instrument can only be bent by $35^{\circ}$. Nonetheless, the advantages in minimally invasive surgery compared to a tool with a rigid shaft are obvious. The function test of the suction showed only a minimal reduction of the suction speed from $5.9 \pm$ $0.15 \mathrm{ml} / \mathrm{s}$ for the elongated wrist joint to $5.7 \pm 0.42 \mathrm{ml} / \mathrm{s}$ for the maximum bent wrist joint. The bending of the wrist has therefore no significant influence on the suction.

The effectivity of the splash protection and the integrated suction has been demonstrated in the phantom tests (see figure 3 ). As displayed in table I, the integrated suction alone could remove between $5.21 \%$ and $63.17 \%$ of the applicated water. For all investigated distances the splash protection increased the ratio between water removed by the integrated suction and applicated water, meaning it made the suction more effective. This effect become stronger with increasing distances. There may be two reasons for this: Firstly, the splash protection prevents a lateral splashing of the water over large distances. Instead, the laterally splashing water is contained at the inside of the splash protection, forms larger drops there and flows down the splash protection. Therefore the splash protection ensures that almost all of the applicated water remains in the sphere of influence of the suction. Secondly, the suction is more effective when the underpressure is stronger. The splash protection reduces the gap through which air can flow from the environment towards the suction to the small gap between splash protection and tissue surface. Thereby the suction can create a stronger underpressure within the splash protection and remove a larger portion of the applicated water. If the underpressure becomes too strong, however, the splash protection can collapse and block both the waterjet and the removal of the applicated water. Although the distance between splash protection and phantom surface was $1 \mathrm{~mm}$ for each test, the measured ratios for the tests with splash protection differ slightly. This may result from two reasons: In some tests (especially for distance $4 \mathrm{~mm}$ ) the water of the waterjet infiltrated the gap between gelatine and the bottom of the bowl. This caused an bulging of the gelatine in the middle of the bowl and therefore reduced the distance between waterjet nozzle and phantom surface. Furthermore, the described collapsing of the splash protection around the tooltip obstructed the suction, which explains the relatively low ratio for using the splash protection at a distance of 10 $\mathrm{mm}$.

In the phantom tests, the target area was even and the nozzle axis remained permanently in an orientation orthogonal to the gelatine surface to improve the comparability of the different test runs. The more uneven the target area is and the more the angle between the nozzle axis and the normal of the 

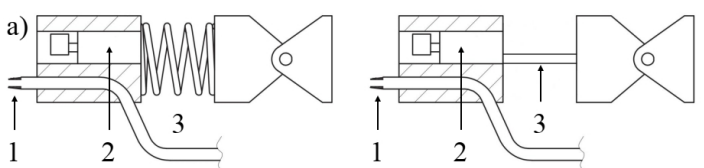

b)
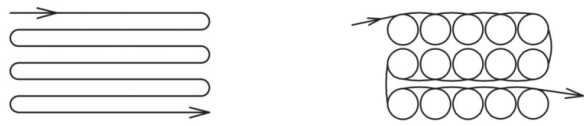

Fig. 4. Low-cost actuation concept for oscilating the tooltip:

a) In addition to the waterjet nozzle 1 the tooltip also integrates a vibration motor 2. A flexible element 3 (which could be a compression spring (left) or a simple spring steel rod (right)) between tooltip and joint allows an oscillation of the tooltip around its position of rest.

b) By integrating adequate actuation means (e.g. a vibration motor and a flexible element) between universal joint and tool tip, the amount and the speed of dissection motions necessary for the abrasion of a predefined area can be reduced. When the actuation at the tool tip performs the high frequent transection motions (represented by the circles in the right sketch), the robot and wrist joints only have to perform less and slower dissection motions (represented by the meandering lines).

target area deviates from 90, the smaller gets the effect of the splash protection. To ensure an effective suction also in such a case, the amplitude of the universal joint movements should be reduced and the nozzle should be moved closer to the target surface to reduce the gap between surface and splash protection.

In the current tool design, the transection motion is created by brushless DC motors in the instrument drive unit and transmitted via tendons to the universal joint at the tooltip. This actuation method was originally designed for teleoperation in minimally invasive surgery, where the required forces are comparatively high (up to $10 \mathrm{~N}$ ) while the required motion frequencies are relatively low (up to $5 \mathrm{~Hz}$ ) [18]. Potentially, the penetration depth of the waterjet and therefore the cutting/abrasion speed can be increased further by a modified tool design allowing higher transection frequencies [20]. One feasible approach is the integration of actuation means for oscillating the tooltip between universal joint and tool tip (compare [23]). While radial or longitudinal piezo elements as described in [23] allow a fast and precise deflection of the tooltip, they are relatively expensive. A low-cost alternative, which could also be applied in a single-use tool, is the integration of a vibration motor and a flexible element at the tool tip (compare figure 4 a)). The vibration motor in the tool tip causes a periodic excitation, whose frequency depends on the motor model and the pulse width modulation (PWM) signal controlling the motor. The flexible element between the motor and the wrist joint of the tool, which can e.g. be a spring steel rod or a compression spring, enables the tooltip to oscillate. When integrating this mechanism in a robotic waterjet tool with a 2-DoF wrist joint as described above, the oscillations caused by the vibration motor can serve as high frequent transection motions. Thus, especially for the abrasion of an area, the necessary amount and the speed of dissection motions, which must be performed by the robot arm and the wrist joint, potentially can be reduced (see figure 4 b)): The
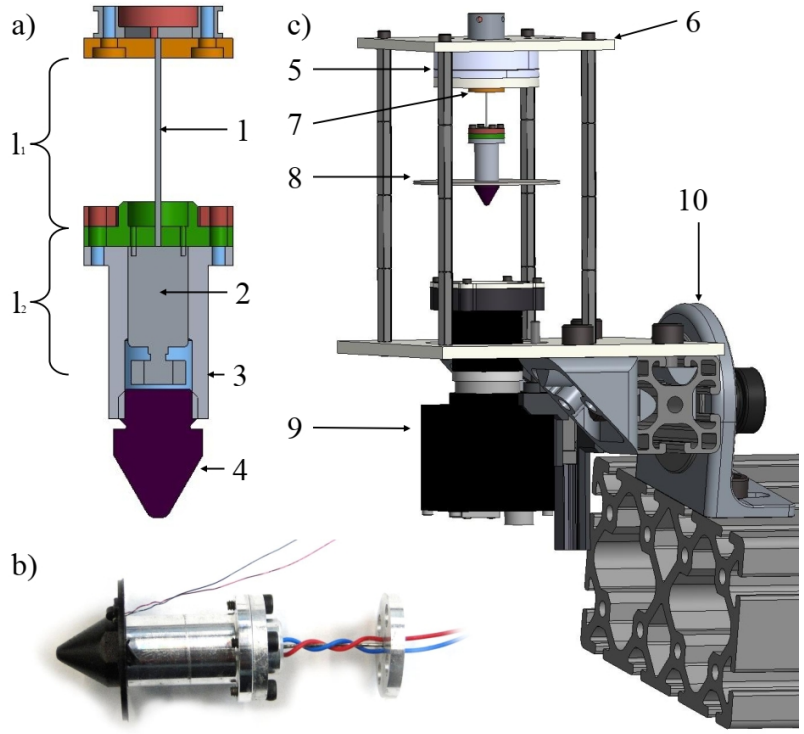

Fig. 5. a) Cross section of the mockup of the tool tip with spring steel rod 1 and vibration motor 2 . The splash protection disc 8 was mounted between motor housing and tip 4.

b) Tool tip mockup used in the function principle tests: The motor cables are twisted around the spring steel rod to minimize their impact on the shape of the tip oscillation. The two thin wires energize the IR-LED for the tracking of the tip motion.

c) Testbed for tool tip mockups of waterjet tools with integrated vibration motor: The FT sensor 5 attached to the mechanical structure 6 and the proximal end of the flexible element 7 measures the forces caused by the vibration motor within the mockup. An IR-LED at a defined position on the splash protection disc 8 is tracked by the monochrome camera 9 to quantify the oscillations of the tooltip. The whole structure can be tilted around the joint 10 to investigate the influence of gravity on the tooltip motions.

number of meandering lines necessary for the abrasion of a predefined area could be decreased by superimposing them with circular motions caused by the vibration motor.

The testbed displayed in figure 5 was designed to investigate the reachable forces and oscillation amplitudes. It contains a 6-DoF force torque (FT) sensor ATI Nano43 [ATI Industrial Automation, NC, USA] and a monochrome camera Prosilica GC 1600H [Allied Vision Technologies, Stadtroda, Germany] in combination with an objective lens Pentax C60636KP 6 mm f/1.4 [Ricoh Imaging, Tokyo, Japan]. The mockup of the tool tip containing a vibration motor VM-0610A3.0 [Elektrotechnik Karl-Heinz Mauz GmbH, Ostfildern, Germany] [24] is connected to the ATI Nano43 via a spring steel rod (diameter $0.5 \mathrm{~mm}$, material X12CrNi177) and an adapter plate. Since the opposite side of the ATI Nano43 is connected to the mechanical structure, the sensor can measure the forces induced by the vibration motor. To measure the oscillations of the tooltip, a SMD IR-LED [Harvatek 1204 HT-110IRAJ, Harvatek Corp., Hsinchu City, Taiwan] is mounted on a defined position on the splash protection disc and its motions are recorded by the Prosilica GC camera below the tooltip mockup. The motor is controled by an Arduino Uno [Arduino AG] and a motor driver Pololu A4990 [Pololu Corporation, Las Vegas, NV, USA].

During the tests, different PWM rates were applied to the 

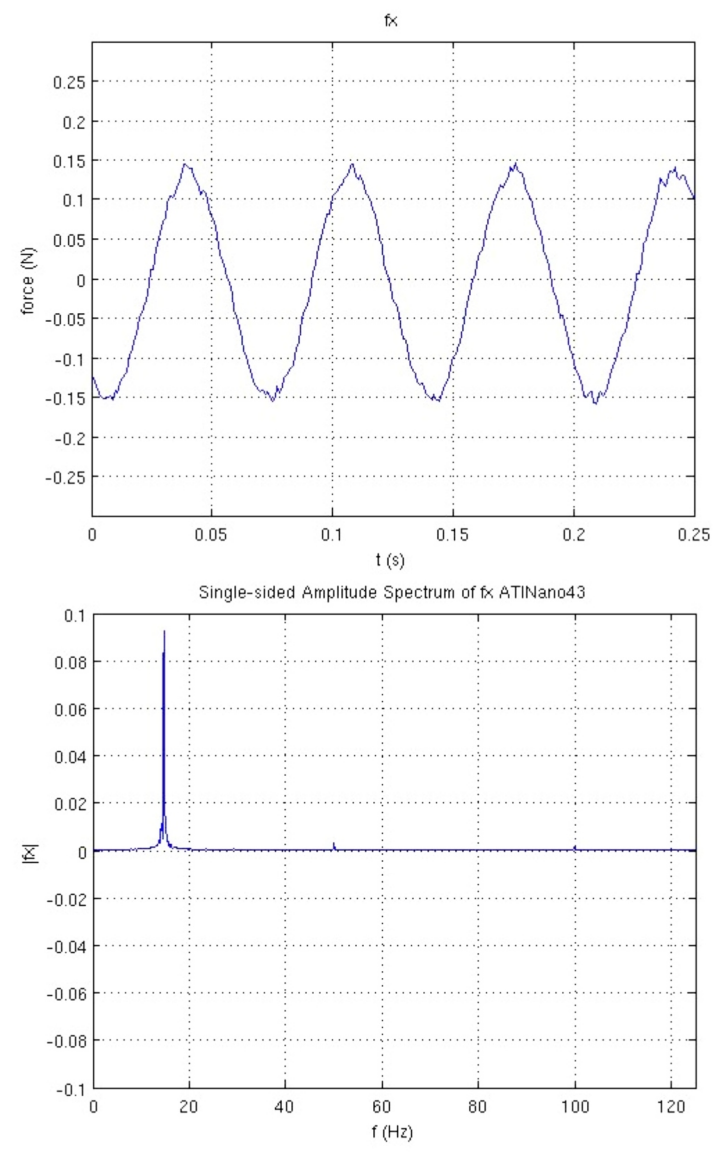

Fig. 6. Measured forces in x-direction of the ATI Nano43 for PWM20: The graph above displays the measured forces over the time, the graph below the result of a Fast Fourier Transformation of the forces. It can be seen, that the forces follow a sinus function with an amplitude of about $0.15 \mathrm{~N}$ and a frequency of about $13.8 \mathrm{~Hz}$.

vibration motor and the resulting forces and motions were recorded. A fast fourier transformation of the forces was performed to determine the rotation frequency of motor. The test results confirmed an increase of the force frequencies with increasing PWM rates. A PWM rate of 20 resulted, for example, in a measured force frequency of $13.8 \mathrm{~Hz}$ and a force amplitude of $0.15 \mathrm{~N}$ (compare figure 6).

As figure 7 shows, the amplitude of the instrument tip motion strongly depended on the excitation frequency. In the test setup, the maximum amplitude of $3.2 \mathrm{~mm}$ was observed for a PWM value of 20. Apart from the PWM value of the motor, the guidance of the LED and vibration motor cables also influenced the oscillation amplitude and shape.

Since the measured force amplitude for PWM 20 was with about $0.15 \mathrm{~N}$ (compare figure 6) similar to the measured thrust of the waterjet reported in [20], the thrust of the waterjet may have significant influence on the shape and amplitude of the oscillation. Additionally, the supply cables of the vibration motor and the waterjet hose act as a parallel spring-damper element and may influence the shape and amplitude of the oscillations significantly if they are not guided properly. And

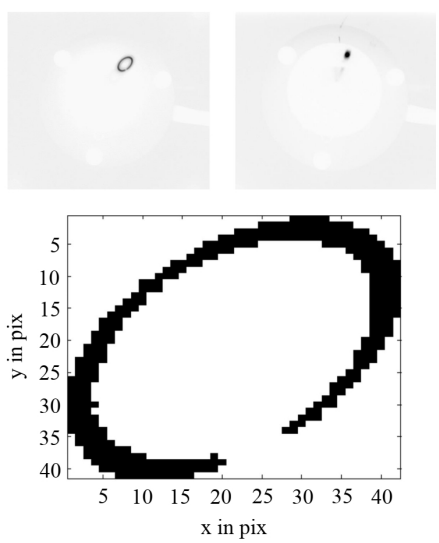

Fig. 7. Camera images showing the oscillation of the tool tip for PWM 20 (top left) and PWM 130 (top right). The exposure time of the camera was adapted to the measured force frequency to reproduce a complete oscillation. A magnified view of the oscillation area (as exemplifiedin the bottom for PWM 20) was used to calculate the amplitude by counting the pixels. In the displayed image 13 pixels correspond to $1 \mathrm{~mm}$.

finally, for strongly tilted operation setups the gravity may have a impact on the oscillation. The center of the elliptical motion of the tool tip is expected to shift in the direction of the gravity vector while the amplitude of the ellipse should not change significantly. While the preliminary tests showed the feasibility of the approach in principle, a more detailled investigation of the above named three issues with a more realistic prototype is necessary. Besides the universal joint, the spring element and the vibration motor, this prototype should integrate a commercial waterjet probe, a technical solution for the suction and provisions for a splash protection. Only after a successful evaluation of this prototype, the construction of a practically applicable, single- or multi-use waterjet instrument with integrated vibration motor can be addressed.

\section{Conclusion}

A waterjet instrument for minimally invasive robotic surgery has been developed. It provides a universal joint with 2 DoF as wrist, an integrated suction and a splash protection to increase the efficiency of the suction and reduce the obstruction of the endoscope optics by waterdrops. Function tests demonstrated the capability of the developed waterjet instrument and the splash protection.

The proposed approach for the design of a low-cost waterjet tool for high oscillation frequencies based on a vibration motor was investigated with regard to reachable forces and oscillation amplitudes. The preliminary tests confirmed the feasibility of the approach in principle, but also revealed a high sensitivity of the tool to external forces resulting from the waterjet thrust, cable stiffness and gravity. A future instrument design must aim at reducing this sensitivity, e.g. by increasing the force amplitudes of the vibration motor.

\section{ACKNOWLEDGMENT}

The authors Christopher Schlenk and Andrea Schwier contributed equally to this work. 


\section{REFERENCES}

[1] D. N. Papachristou and R. Barters, "Resection of the liver with a water jet," British journal of Surgery, vol. 69, no. 2, pp. 93-94, 1982.

[2] Y. Une, J. Uchino, T. Horie, Y. Sato, K. Ogasawara, A. Kakita, and F. Sano, "Liver resection using a water jet," Cancer chemotherapy and pharmacology, vol. 23, no. 1, pp. 74-77, 1989.

[3] R. Izumi, K. Yabushita, K. Shimizu, M. Yagi, A. Yamaguchi, K. Konishi, T. Nagakawa, and I. Miyazaki, "Hepatic resection using a water jet dissector," Surgery today, vol. 23, no. 1, pp. 31-35, 1993.

[4] H. Rau, G. Meyer, T. Cohnert, H. Schardey, K. Jauch, and F. Schildberg, "Laparoscopic liver resection with the water-jet dissector," Surgical endoscopy, vol. 9, no. 9, pp. 1009-1012, 1995.

[5] H. Rau, E. Buttler, G. Meyer, H. Schardey, and F. Schildberg, "Laparoscopic liver resection compared with conventional partial hepatectomya prospective analysis." Hepato-gastroenterology, vol. 45, no. 24, pp. 2333-2338, 1998.

[6] H. Rau, A. Duessel, and S. Wurzbacher, "The use of water-jet dissection in open and laparoscopic liver resection," $H P B$, vol. 10, no. 4, pp. 275 280, 2008.

[7] S. Palmier and C. Trial, "Use of high-pressure waterjets in wound debridement," in Surgery in wounds. Springer, 2004, pp. 72-76.

[8] B. Oosthuizen, T. Mole, R. Martin, and J. G. Myburgh, "Comparison of standard surgical debridement versus the versajet plus hydrosurgery system in the treatment of open tibia fractures: a prospective open label randomized controlled trial," International journal of burns and trauma, vol. 4, no. 2, pp. 53-58, 2014.

[9] R. Basting, S. Corvin, C. Antwerpen, N. Djakovic, and D. Schmidt, "Use of water jet resection in renal surgery: early clinical experiences," European urology, vol. 38, no. 1, pp. 104-107, 2000.

[10] F. Köckerling, C. Yildirim, J. Rose, H. Scheidbach, and P. Geers, "Total mesorectal excision with the water-jet-dissection. technique and results," Techniques in coloproctology, vol. 8, no. 1, pp. 217-225, 2004.

[11] S. Corvin, W. Sturm, E. Schlatter, A. Anastasiadis, M. Kuczyk, and A. Stenzl, "Laparoscopic retroperitoneal lymph-node dissection with the waterjet is technically feasible and safe in testis-cancer patient," Journal of endourology, vol. 19, no. 7, pp. 823-826, 2005.

[12] J. Oertel, M. Gaab, and J. Piek, "Waterjet resection of brain metastasesfirst clinical results with 10 patients," European Journal of Surgical Oncology, vol. 29, no. 4, pp. 407-414, 2003.

[13] J. Oertel, W. Wagner, J. Piek, H. Schroeder, and M. Gaab, "Waterjet dissection of gliomas-experience with 51 procedures," min-Minimally Invasive Neurosurgery, vol. 47, no. 03, pp. 154-159, 2004.

[14] D. Keiner, M. R. Gaab, V. Backhaus, J. Piek, and J. Oertel, "Water jet dissection in neurosurgery: an update after 208 procedures with special reference to surgical technique and complications," Operative Neurosurgery, vol. 67, no. suppl_2, pp. ons342-ons354, 2010.

[15] Versajet II Hydrosurgery System, Website, Smith \& Nephew Inc. 2012, available: http://www.smith-nephew.com/key-products/advancedwound-management/versajet/.

[16] The versatility of hydrosurgery: ERBEJET2 with hybrid instruments, Website, Erbe Elektromedizin $\mathrm{GmbH}, 2016$, available: https://de.erbemed.com/de-en/products/hydrosurgery/product/erbejet- 2 .

[17] debritom + Instruction for use, Website, Medaxis AG, 2017, available: https://www.medaxis.ch/en.html.

[18] S. Thielmann, U. Seibold, R. Haslinger, G. Passig, T. Bahls, S. Jörg, M. Nickl, A. Nothhelfer, U. Hagn, and G. Hirzinger, "Mica-a new generation of versatile instruments in robotic surgery," in IEEE/RSJ International Conference on Intelligent Robots and Systems (IROS) 2010. IEEE, 2010, pp. 871-878.

[19] T. Bahls, F. A. Fröhlich, and A. O. Albu-Schäffer, "Towards robot guided waterjet surgery," in Proceedings of CURAC 2013, 2013, pp. 80-84.

[20] T. Bahls, F. A. Fröhlich, A. Hellings, B. Deutschmann, and A. O. Albu-Schäffer, "Extending the capability of using a waterjet in surgical interventions by the use of robotics," IEEE Transactions on Biomedical Engineering, vol. 64, no. 2, pp. 284-294, 2017.

[21] J. Jussila, A. Leppäniemi, M. Paronen, and E. Kulomäki, "Ballistic skin simulant," Forensic science international, vol. 150, no. 1, pp. 63-71, 2005.

[22] D. S. Schoeb, J. Klodmann, D. Schlager, P. F. Müller, A. Miernik, and T. Bahls, "Robotic waterjet wound debridement - workflow adaption for clinical application and systematic evaluation of a novel technology," PLOS ONE, vol. 13, no. 9, pp. 1-14, 09 2018. [Online]. Available: https://doi.org/10.1371/journal.pone.0204315
[23] R. Kühner and L. Mitzlaff, "Fluid-surgical instrument with variable spray image," Erbe Elektromedizin $\mathrm{GmbH}$, 2014, uS 20140107683A1. [Online]. Available: https://patents.google.com/patent/US20140107683A1/en

[24] VM-0610A3.0, Website, EKULIT Elektrotechnik KarlHeinz Mauz GmbH, available: https://www.ekulit.de/wpcontent/uploads/datasheets/860210 